This is the author's version of the work. It is posted here by permission of the AAAS for personal use, not for redistribution. The definitive version was published in Science on 25 May 2018:

Vol. 360, Issue 6391, pp. 861-862 DOI: 10.1126/science.aar8480

\title{
Disparities in science literacy
}

Cognitive and socioeconomic factors don't fully explain gaps

Nick Allum ${ }^{1}$, John Besley ${ }^{2}$, Louis Gomez ${ }^{3,4}$, Ian Brunton-Smith ${ }^{5}$

1. Department of Sociology, University of Essex, Colchester, UK.

2. Department of Advertising and Public Relations, Michigan State University, East Lansing, MI, USA.

3. Department of Education, University of California, Los Angeles, CA, USA.

4. Carnegie Foundation for the Advancement of Teaching, Stanford, CA, USA.

5. Department of Sociology, University of Surrey, Guildford, UK

Much is known about how adult science literacy varies internationally and over time, and about its association with attitudes and beliefs. However, less is known about disparities in science literacy across racial and ethnic groups (1). This is particularly surprising in light of substantial research on racial and ethnic disparities in related areas such as educational achievement, math and reading ability (2), representation in science, technology, engineering, and math (STEM) occupations (3), and health literacy (4). Given the importance of science literacy to securing and sustaining many jobs, to understanding key health concepts to enhance quality of life, and to increasing public engagement in societal decision-making (5), it is concerning if the distribution of science literacy is unequally stratified, particularly if this stratification reflects broader patterns of disadvantage and cultural dominance as experienced by minorities and educationally underserved populations. We describe here such disparities in science literacy in the United States and attempt to explain underlying drivers, concluding that the science literacy disadvantage among black and Hispanic adults relative to whites is only partially explained by measures of broader, foundational literacies and socioeconomic status (SES).

The main source of evidence about U.S. patterns and trends in science literacy is the National Science Board's Science and Engineering Indicators (SEI) survey module (3), administered biennially since 2006 to a subsample of respondents for the General Social Survey (GSS), a high-quality biennial survey that seeks to provide a representative picture of American adults (aged 18 or older). Science literacy is captured by questions covering basic scientific facts and processes, but subgroup analysis is only presented in SEl for gender, age, education, and income because sample sizes do not permit more granular analysis. Research using different questions (6) found that white Americans score more highly than blacks and Hispanics, although sample sizes for black and Hispanic groups were relatively low and only bivariate analysis was presented. Other work (7) found that black Americans reported lower confidence in science, even after adjusting for attitudinal and demographic factors, though this study did not look at science literacy.

In contrast to the sparse research on race and adult science literacy, there is voluminous evidence of racial inequalities in educational measures of children's science knowledge (8). Moving beyond narrow science literacy to health literacy and foundational reading literacy, we see similar stratified patterns where white Americans do better than blacks and Latinos, with substantial variation across SES groups (9).

In the present study, our first objective is to examine racial and ethnic disparities in science literacy among adults in the United States. We estimate these by combining data from six waves of the GSS between 2006 and $2016(n=2339)$. We take it as axiomatic that the explanation for such disparities must be found in socially determined factors that fall differentially on different groups. Our second objective is therefore to investigate plausible factors, including demographics, foundational literacy, attitudes, and access to information that could account for such disparities [see supplementary materials (SM) for details on all data and analyses].

The GSS science survey module includes multiple choice (mostly true/false) questions about science content and process, along with open-ended questions. We regard these questions as indicators of the broader construct of science literacy. To measure foundational literacy, we rely on a well- 
established measure of verbal ability, Wordsum, that has been included in the GSS since 1974 (10). We use a standard set of demographic controls, including gender, birth cohort, geographical region, education, income, and religion. We also employ the following covariates that we hypothesize could account for between-group literacy differences.

We use a particularly rich measure of SES, the Cambridge Social Interaction and Stratification (CAMSIS) scale (11), that represents differences in status, prestige, and economic advantage, based on respondents' occupational groups. This measure is useful as it reflects the kinds of personal networks, social class, and cultural milieu, in which views about science develop and which may overlap with racial inequality. Further, we might surmise that a largely white teaching force has often failed to understand contexts of social life and interests of black and Hispanic students, to connect those interests to scientific phenomena, and to support scientific literacy about the phenomena. This neglect may lead to different levels of science literacy even given equivalent formal qualifications (12).

Some minority groups express less trust and confidence in science compared to whites (7). Low confidence in science arguably could lead to lack of science engagement in various settings, thus lower knowledge scores. We therefore include measures that ask people how much "confidence" they have in the "scientific community" and how positive they are about science.

One potential benefit of the internet would be in reducing the gap between the information-rich and information-poor. Yet, knowledgeable individuals are often able to acquire information more effectively, so the internet may exacerbate knowledge gaps (13). We include a question on whether respondents have sought science information on the internet.

Pooling the samples across all years yields a mean science literacy quiz score for whites of 8.6 (out of a maximum possible 13), Hispanics 6.8, and blacks 6.5. A one-way analysis of variance shows statistically significant differences between groups $(F=283$; $d f=3)$. The overall mean for all groups combined is 8.0 with a standard deviation of 2.7 ; the average difference between whites and the two racial and ethnic groups is quite substantial, at around two-thirds of a standard deviation.

We tried to gain better understanding of these disparities by adjusting for potential confounding factors. We fit several multivariate ordinary least squares regression models with science knowledge as the dependent variable. The first model included indicators for race and ethnicity alongside demographic variables: sex, birth cohort, region of residence, college education, and income. We also include SES and number of science courses taken at high school or college level. If science literacy disparities are due to these factors, then statistically adjusting for them should attenuate or remove any residual differences among the groups. In the second model, we added our measure of foundational literacy. If this eliminates race or ethnicity differences, it would support the hypothesis that broader literacy disparities lie behind science literacy disparities. In the third model, we added attitude toward science, confidence in science, and science internet use, to see if these more proximal features of orientation to science and technology might lie behind science literacy differences.

Even after adjusting for demographic variables, science knowledge disparities are only partially reduced. There is still around a 1.5-point difference in the average scores of black and white Americans [see the figure, model 1 (blue)]. The gap for Hispanics is narrower, but their mean is still about a point lower than that for whites. Most of the other included predictors are significantly associated with science literacy and consistent with extant research (fig. S1 and table S1). Model 1 accounts for about $20 \%$ of the variance in science literacy.

Adding foundational literacy to the model shrinks the coefficients for both minority groups. Residual gaps are just over one point for blacks and a half point for Hispanics [see the figure, model 2 (red)]. This model accounts for just under $22 \%$ of the variance. The hypothesis that disparities in foundational literacy account for gaps in science literacy receives some support, but there is much left to explain. Adding behavioral and psychological variables increases the amount of variance explained by the model to $30 \%$, but we see little change in the race and ethnicity coefficients [see the figure, model 3 (green)]. Race and ethnicity continue to matter even when comparing the science literacy of people with similar science attitudes.

Overall, disparities in science literacy cannot be straightforwardly "explained" by intergroup differences in the levels of our measured characteristics. We performed a decomposition analysis and 
found that whereas around one-third of the variation in knowledge scores is explained by the independent variables, only about half of the total race and ethnicity gaps are explained by these observables. For both blacks and Hispanics, differences in foundational literacy compared to whites are the most important of the observable influences on the size of the disparity. The remaining, unexplained, portion of the gap must in large part be driven by variables not in our models.

Translating confidence in, and positive attitudes toward, science into higher science literacy appears to be less common for blacks and Hispanics than for whites, for unclear reasons.

Change in science knowledge score per unit change in independent variables

Unstandardized regression coefficients. Model $1=$ demographic variables; model $2=$ model $1+$ foundational literacy; model $3=$ model $2+$ behavioral/psychological variables. Effect of black/Hispanic/other is compared to white. Effect of college or above is compared to less than college. The zero line means no effect. See full results in the SM.

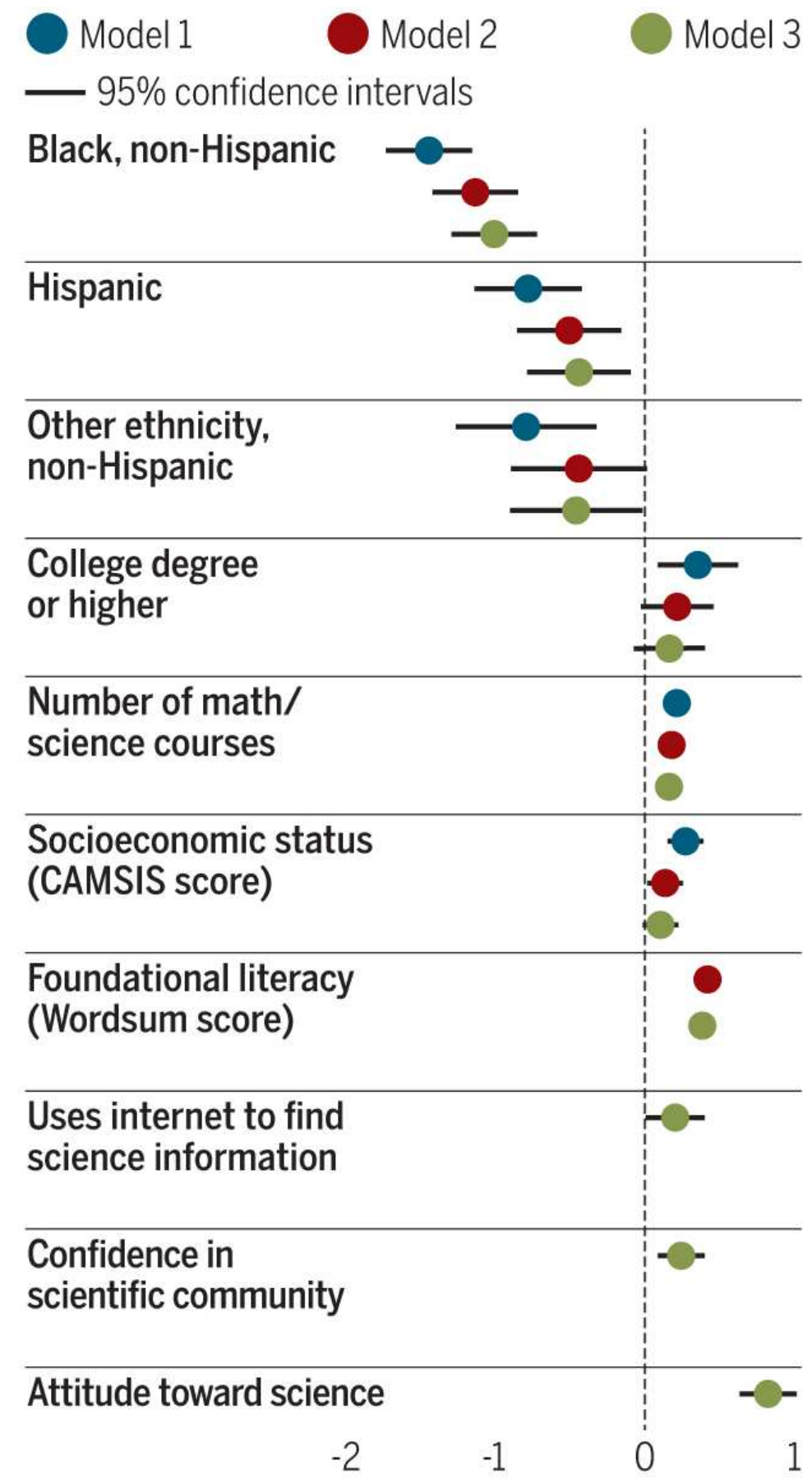


A principal question we wanted to address was the extent to which ethnic and racial inequalities in science literacy are simply reflections of well-established disparities in more fundamental axes of disadvantage, including broader foundational literacies. They are not. When we compared whites with black and Hispanic respondents who hold similar attitudes toward science and have the same degree of confidence in its institutions, we still find persistent disparities in science literacy. We do not claim to have captured all of these disadvantages in our analysis, as our variables are measured with error and are relatively broad-brush, but we have at least included key dimensions.

This analysis invites the question as to what could be responsible for the remaining gaps. It may be that the specific knowledge questions asked or the survey response context favors whites, but, more important, we suspect that our education measures mask considerable heterogeneity in the experiences of children, young people, and adults of different races and ethnicities. Graduating from high school, earning a college degree, or taking a science class can consist in a wide variety of experiences, some of which are likely correlated with race and ethnicity. As recently as the early 1970s, black and Hispanic children were much more likely to attend schools funded at a lower than average rate and intentionally segregated by ethnicity, and such segregation has continued de facto to varying degrees (14). Microsocial experiences of nondominant groups in any learning environment-for example, stereotype threat and racial microaggressions-can shape learning experiences (15). Although we adjust for educational qualifications, we do not capture differences in the quality of education experienced by blacks and Hispanics.

This suggests that educational interventions need to measure, and target, not just the quantity of instruction and formal qualifications, as we do here, but also quality. We may also be able to craft training and public awareness campaigns to help scientists, teachers, and employers to be more sensitive to the subtle manifestations of bias. Whatever the remedy, ignoring science literacy disparities among underserved groups does not serve science or society well.

\section{References}

1. National Academies of Sciences, Engineering, and Medicine, Science Literacy: Concepts, Contexts, and Consequences (National Academies Press, Washington, DC, 2016).

2. G. Kena et al., The Condition of Education 2014 (NCES 2014-083, U.S. Department of Education, National Center for Education Statistics); http://nces.ed.gov/pubs2014/2014083.pdf (2014).

3. National Science Board, "Science and Engineering Indicators 2016" (National Science Foundation, Arlington, VA, 2016).

4. R. V. Rikard et al., BMC Public Health 16, 975 (2016).

5. W. K. Hallman, Handbook of the Science of Science Communication (Oxford Univ. Press, 2017), pp. 61-72.

6. Pew Research Center, A Look at What the Public Knows and Does Not Know About Science (Washington, DC, 2015).

7. E. Plutzer, Bull. Sci. Technol. Soc. 33, 146 (2013).

8. National Center for Education Statistics (2012), The Nation's Report Card: Science 2011 (NCES 2012-465). Institute of Education Sciences, U.S. Department of Education (Washington, DC, 2011).

9. C. C. Cutilli, I. M. Bennett, Orthop. Nurs. 28, 27 (2009).

10. N. Malhotra, J. A. Krosnick, E. Haertel, The psychometric properties of the GSS Wordsum vocabulary test. GSS Methodological Report 11 (NORC, 2007). 
11. K. Prandy, Sociology 24, 629 (1990).

12. S. D. Museus, R. T. Palmer, R. J. Davis, D. Maramba, Racial and Ethnic Minority Student Success in STEM Education: ASHE Higher Education Report, Volume 36, Number 6 (Wiley, 2011).

13. L. Su, M. A. Cacciatore, D. A. Scheufele, D. Brossard, M. A. Xenos, Sci. Commun. 36, 352 (2014).

14. L. Darling-Hammond, Handbook of Research on Multicultural Education, J. A. Banks, Ed. (Jossey-Bass, San Francisco, 2001), pp. 465-583.

15. S. M. Quintana, L. Mahgoub, Theory Into Practice 55, 94 (2016).

\section{Supplementary Materials}

www.sciencemag.org/content/360/6391/861/suppl/DC1 